\title{
UNA APROXIMACIÓN TEÓRICA A LA DESERCIÓN ESTUDIANTIL UNIVERSITARIA
}

\section{A THEORETICAL APPROACH TO THE COLLEGE STUDENT DROP OUT}

\author{
Héctor Ernesto Viale Tudela* Universidad \\ Peruana de Ciencias Aplicadas, Perú
}

\section{RESUMEN}

La deserción estudiantil universitaria es uno de los mayores problemas con el que se enfrenta el sistema de educación superior. Las autoridades de las universidades han venido presentando gran preocupación debido a que ante el aumento en la demanda de la educación superior, el número de alumnos que logra culminar sus estudios superiores no es el esperado, evidenciándose el abandono de un gran número de estudiantes en los primeros semestres. Esto genera problemas financieros en las universidades. La finalidad de este artículo es mostrar, luego de una exhaustiva revisión bibliográfica, los modelos existentes en relación con la deserción estudiantil en la universidad y responder las cuestiones que giran en torno a este tema.

Palabras clave: deserción, deserción estudiantil, permanencia, retención

\begin{abstract}
College student dropout is one of the greatest problems faced by the higher education system. University authorities have been showing great concern because, in spite of the increase in demand for higher education, the number of students that complete their education is not as expected, demonstrating that a large number of students abandons school, mainly during the initial semesters. This generates financial problems to the universities. This paper aims to show, after a comprehensive literature review, existing models in connection with college student dropout and answer the questions to this regard.
\end{abstract}

Keywords: desertion, student dropout, student retention 
¿A qué se debe la deserción estudiantil universitaria?

¿Por qué el rendimiento académico, durante los primeros ciclos, de la mayoría de los alumnos recién ingresados a la universidad es menor que en la escuela a pesar de haber sido buenos alumnos en la misma? ¿Por qué los alumnos recién ingresados a la universidad, la abandonan en su mayoría? ¿Es responsabilidad de la universidad? ¿Qué podemos hacer desde la universidad para revertir esta situación? Si articulamos las actividades de las distintas unidades que reciben a los alumnos recién ingresados a la universidad, ¿lograremos disminuir la deserción? ¿Las autoridades universitarias creen en los programas de inserción a la universidad?

Sabemos, los que nos movemos en el mundo de la docencia universitaria, que los resultados académicos de la mayoría de los alumnos recién ingresados a la universidad no son los deseados. Existe una brecha académica entre lo que se enseña en las escuelas y lo que se exige en las universidades. Este no es un problema nuevo ni exclusivo del ámbito académico ni propio del Perú. Es más bien un viejo problema que tiene muchas variables y que, además, se da en todo el mundo. El porcentaje de alumnos desaprobados en una o más asignaturas del primer ciclo es elevado, lo cual lleva a que varios de ellos se matriculen, nuevamente, en las mismas asignaturas. A pesar de esto, muchos vuelven a desaprobar y terminan abandonando la universidad, con lo que el número de alumnos con educación superior incompleta aumenta en el país (viéndose perjudicado el mismo estudiante y su familia) y, adicionalmente, el presupuesto de las universidades también se ve afectado.

Según González y Girón (2005), la deserción estudiantil tiene consecuencias sociales, emocionales y económicas no solo en el propio estudiante sino en su entorno más cercano. Adicionalmente, quienes no concluyen sus estudios son subempleados y no obtienen los ingresos económicos deseados (Díaz, 2008).

Este viejo problema de la deserción tiene muchas variables, y las podemos dividir en aquellas que pertenecen al ámbito pedagógico pero también en aquellas que pertenecen al ámbito no pedagógico (Tejedor \& García-Valcárcel, 2007), como las variables académicas, psicológicas, socio-familiares y de identificación, que influyen en el rendimiento académico de los alumnos recién ingresados a la universidad. Atendiendo estos dos tipos de variables (las pedagógicas y las no pedagógicas), cada universidad ha diseñado sus propios programas para facilitar la adaptación a la vida universitaria de los estudiantes nuevos pero, en la mayoría de los casos, estos programas pertenecen a departamentos o áreas académicas distintas, con estructuras organizacionales diferentes; con lo cual la orientación al alumno recién ingresado se hace desde distintas concepciones. Esto, en vez de ayudar al estudiante, termina por confundirlo más y no se alcanza el objetivo de facilitar su inserción a la universidad.

El estudio de la deserción estudiantil en la universidad, para su posterior control, es muy importante, pues la tasa de deserción se está empezando a considerar como un indicador de la calidad de la gestión universitaria: de hecho, la tasa de abandono figura como indicador de calidad en numerosos modelos de evaluación de la institución universitaria (MEC: Catálogo de indicadores del sistema universitario público español), y como indicador en los ranking de universidades (Yorke, 1998, como se citó en Cabrera, Bethencourt, Álvarez Pérez \& 
Gónzalez 2006). Actualmente, en el Perú, la deserción estudiantil universitaria es tomada como un indicador para que las universidades peruanas puedan acreditarse (Coneau, 2010).

Según Cabrera et al. (2006), las tasas de deserción estudiantil son un indicador de baja calidad, pues se entiende que la universidad no hizo lo necesario para que los estudiantes terminaran su carrera.

\section{¿Cómo definimos la deserción estudiantil universitaria?}

No existe una única definición para el concepto de deserción estudiantil universitaria. Según el Diccionario Enciclopédico de Ciencias de la Educación (Picardo, O., Escobar, J., \& Balmore, R., 2004), deserción es el acto deliberado o forzado mediante el cual el estudiante deja su aula o centro educativo.

Por otro lado, Tinto (1975) afirma que existe una gran variedad de comportamientos llamados deserción, pero adicionalmente señala que este concepto no debe abarcar todos los abandonos de estudio, así como que no necesariamente todas las deserciones estudiantiles merecen la intervención de la institución.

En la Universidad Autónoma Metropolitana, México, se define a la deserción como el hecho mediante el cual un alumno interrumpe de manera voluntaria o involuntariamente sus estudios para siempre, sin haber cubierto en su totalidad el plan de estudios de la carrera respectiva. A fin de hacer operativa esta definición, ellos identifican tres modalidades de desertores (Durán y Díaz, 1990): 1. La deserción voluntaria, cuando el alumno realiza el procedimiento establecido por la administración escolar denominado Baja Definitiva para darse de baja voluntariamente.
2. La deserción incurrida, cuando el alumno viola las disposiciones reglamentarias de la institución.

3. La deserción potencial, cuando el alumno no ha realizado trámites académicoadministrativos durante siete o más trimestres consecutivos y tiene menos de 35 créditos académicos para las licenciaturas impartidas por las Unidades Azcapotzalco e Iztapalapa o menos de 10 para las de la Unidad Xochimilco, en virtud de que el número señalado de las UEA corresponde a un porcentaje menor al $80 \%$ del total de las contempladas en los planes de estudio.

Adicionalmente, Tinto (1982) define el concepto de deserción como una situación a la que se enfrenta un estudiante cuando sus proyectos educativos no logran concretarse. Luego, Tinto (1982) concluye que podemos considerar como desertor a aquel estudiante que no presenta actividad académica durante tres semestres académicos consecutivos. En algunas investigaciones, a este comportamiento se le denomina 'primera deserción' (first drop-out), ya que no se puede determinar si, pasado este periodo de tiempo, el individuo retomará o no sus estudios o si decidirá iniciar otro programa académico (Tinto, 1989).

Por otro lado, Tinto (1989) afirma que ninguna definición de deserción logra capturar en su totalidad este fenómeno y deja en manos de los investigadores la elección de la definición que mejor se ajuste a la investigación que lleven a cabo.

Según Castaño, Gallón, Gómez y Vásquez (2004), se pueden reconocer dos tipos de abandonos en los estudiantes universitarios: con respecto al tiempo y al espacio. 
Con respecto al tiempo, la clasifican a su vez en:

1. Deserción precoz, cuando el estudiante que, habiendo sido admitido por la universidad, no se matricula.

2. Deserción temprana, cuando el estudiante abandona sus estudios en los cuatro primeros semestres de la carrera.

3. Deserción tardía, cuando el estudiante abandona los estudios en los últimos seis semestres, es decir, a partir del quinto semestre en adelante.

Por su parte, la deserción con respecto al espacio la dividen en:

1. Deserción interna o del programa académico, se refiere al estudiante que decide cambiar su programa académico por otro que ofrece la misma institución universitaria.

2. Deserción institucional, es el caso en el cual el estudiante abandona la universidad para matricularse en otra, $y$

3. La total deserción del sistema educativo.

Simpson (2004), siguiendo la línea de Tinto (1989), afirma que el estudio de la deserción de estudiantes universitarios es extremadamente complejo y que ninguna definición de deserción logra capturar completamente la complejidad del problema.

Por otro lado, según los indicadores del Contrato Programa que la Universidad Autónoma de Barcelona tiene firmado con la Generalitat de Cataluña, definen el abandono como alumnos matriculados en alguna titulación que durante dos años consecutivos no vuelven a formalizar la matrícula (Vivas, 2005).

Para Cabrera et al. (2006), el abandono de estudios o deserción estudiantil son los términos que los castellano-parlantes hemos adoptado para denominar a una variedad de situaciones identificadas en el proceso educativo del estudiantado con un denominador común, detención o interrupción de estudios iniciados antes de finalizarlos. Esta categoría, según Cabrera et al. (2006), incluye:

- Abandono involuntario (por incumplimiento administrativo o violación de reglamentos);

- Dejar la carrera para iniciar otra en la misma institución;

- Dejar la carrera para iniciar otra en otra institución;

- Dejar la universidad e irse a otra para completar estudios iniciados;

- Renunciar a la formación universitaria para iniciar itinerarios formativos fuera de la universidad o incorporarse al mundo laboral;

- Interrumpir la formación con la intención de retomarla en el futuro; $y$

- Otras posibilidades.

Las estadísticas universitarias suelen identificar como caso de abandono al estudiante que ha iniciado estudios y que, antes de finalizarlos, no se vuelve a matricular por dos semestres académicos consecutivos. Dentro de esta amplia categoría, encontramos situaciones que no pueden catalogarse de abandono de estudios y, mucho menos, de abandono de la formación universitaria, como son los estudiantes que completan su formación en otrainstitución (Cabrera etal., 2006).

A su vez, Díaz (2008) considera como deserción al hecho de que si después de tres ciclos académicos el estudiante ya no se vuelva a matricular en ella.

Por otro lado, Himmel (2002) define la deserción como voluntaria (cuando el estudiante renuncia a la carrera o no informa a la institución cuando abandona la universidad) y como involuntaria (como consecuencia de una decisión institucional fundada en sus reglamentos vigentes). 
Díaz (2008) señala que a pesar de que la definición de deserción estudiantil está en discusión, existe consenso en definirla como un abandono voluntario que puede ser explicado por diferentes categorías de variables: socioeconómicas, individuales, institucionales y académicas.

\section{¿En qué etapa de los estudios universitarios sucede el mayor porcentaje de abandono?}

Corominas (2001), en un estudio realizado en la universidad de Girona, concluye "que las deserciones se producen mayormente en primero, debido a elecciones inadecuadas de la carrera a elegir, bajo rendimiento académico, no haber aprobado el mínimo de créditos, asignaturas poco motivadoras, y poco esfuerzo y compromiso con el estudio". (p. 130).

Por su lado, en su Trabajo presentado en el Encuentro Internacional de Deserción Estudiantil en Educación Superior en Bogotá, Colombia, J. Vivas (2005) señala que a nivel de la Universidad Autónoma de Barcelona, alrededor del $60 \%$ del abandono se produce en el primer ciclo.

En general, las estadísticas hablan de una media de $26 \%$ de abandonos en el primer año. Algunos estudios concretos, realizados en universidades españolas, sitúan las cifras entre un 15 y un $20 \%$ (De Miguel y Arias, 1999). Las distintas estadísticas recogidas en los informes emitidos en el marco de los Planes Nacionales de Evaluación, en España, sitúan porcentajes medios de un $16 \%$ entre el alumnado de primer año (Cabrera et al., 2006).

En un trabajo de investigación llevado a cabo por la UNESCO (Rivera, E., Roca, H., Echart, B., Alfaro, E., López, A., Farfán, S., Barrera, B.) en el año 2005 en coordinación con la IESALC, en la universidad Juan Misael Saracho de Bolivia, se ha podido concluir como relevante que la mayor frecuencia de abandonos (deserción) ocurre después del segundo año de estudios.

\section{La aproximación teórica}

A partir de los aportes de Braxton, ShawSullivan y Johnson (1997), se proponen cinco grandes modelos teóricos para clasificar los enfoques sobre deserción y retención, según el énfasis asignado a las tres variables explicativas fundamentales; a saber: personales, familiares o institucionales. En razón de ello, se identifican las categorías psicológica, sociológica, económica, organizacional y la interaccionista.

Bethencourt, Cabrera, Hernández, Álvarez y González (2008) señalan que si se ordenan las variables analizadas según el grado de asociación percibido en el abandono universitario, se tendría que el orden sería el siguiente: (a) características psicológicas; (b) estrategias y actividades de estudio; (c) características del profesorado; (d) características de la titulación.

Las investigaciones de Bethencourt et al. (2008), han permitido demostrar la importancia de ciertas características psicológicas del estudiantado universitario en la terminación de sus estudios. Parece ser que la persistencia para acabar la titulación a pesar de los obstáculos, la motivación hacia la titulación cursada, la capacidad de esfuerzo en favor de logros futuros, el ajuste entre las capacidades del alumnado y las exigencias de la titulación, y la satisfacción con la titulación cursada son garantías de éxito académico.

Gran parte de la literatura referida a la permanencia de los estudiantes en la universidad se desarrolla sobre la base de dos principales teorías sociológicas: el modelo de integración del estudiante (Student 
Integration Model) Spady (1971) de Tinto (1975) y el modelo de desgaste del estudiante (Student Attrition Model) de Bean y Metzner (1985). El primero de los modelos explica que, dadas todas las demás variables constantes, un mayor grado de integración del estudiante en el ambiente académico y social contribuye a un mayor grado de compromiso institucional y esto afecta directamente a la decisión del alumno de permanecer o desertar. El segundo modelo atribuye mayor importancia relativa a los factores externos a la institución. Cabrera, Bethencourt, Álvarez y González (2006) consideran que si bien estos modelos parecieran ser opuestos, en realidad existe complementariedad entre ellos y a su vez superposición en algunos aspectos. Es así como proponen un modelo integrado que enfatiza los procesos sociológicos y psicológicos del comportamiento de la persistencia del alumno en la universidad (Giovagnoli, Paula; 2002).

A continuación, se describen algunas teorías sobre la deserción que permiten enfocar el problema desde distintas perspectivas.

A la hora de valorar el abandono académico en la enseñanza superior, se han propuesto diferentes modelos y teorías explicativas, que Cabrera et al. (2006) agrupan en cinco grandes enfoques (que nosotros hemos llamado categorías): psicopedagógico, adaptación, estructural, economicista e interaccionista.

Categoría 1.- Enfoque psicológico. La principal característica se refiere a los rasgos de personalidad que diferencian a los alumnos que terminan sus estudios respecto a los que no lo hacen. La propuesta de Fishbein y Ajzen (1975) es uno de los primeros modelos que aparece en la literatura sobre el tema. Ellos proponen la Teoría de la Acción Razonada y muestran que el comportamiento está influido significativamente por las creencias y actitudes. De esta forma, la decisión de desertar o continuar en un programa académico está determinada por:

- Las conductas previas,

- Las actitudes sobre la deserción y persistencia, $\mathrm{y}$

- Normas subjetivas acerca de estas acciones, las que generan "una intención conductual", que es un comportamiento definido.

Este modelo asume la deserción como el debilitamiento de las intenciones iniciales y la retención como un fortalecimiento de las mismas.

“...en las percepciones del estudiantado universitario, las variables del alumno son vistas como más relevantes que las variables del contexto en el abandono de sus estudios universitarios"(Bethencourtetal,2008,p.610).

A continuación, en el Diagrama 1, podemos ver un esquema del modelo de Fishbein y Ajzen (1975).

Attinasi (1986) amplía el modelo expuesto con la idea de que la persistencia o la deserción se ven influidas por el análisis que hacen los estudiantes universitarios en relación con su vida universitaria. De esta manera, efectúan una evaluación del resultado de dicho análisis para su posterior permanencia o abandono.

Eccles y Wigfield (2002) señalan que el rendimiento académico previo a los estudios universitarios influye en el desempeño futuro del estudiante.

Por su lado, Ethington (1990) encontró que el nivel de aspiraciones tenía un efecto directo sobre los valores. Además, pudo observar que las expectativas de éxito estaban explicadas por el autoconcepto académico y la percepción de las dificultades de los estudios. 


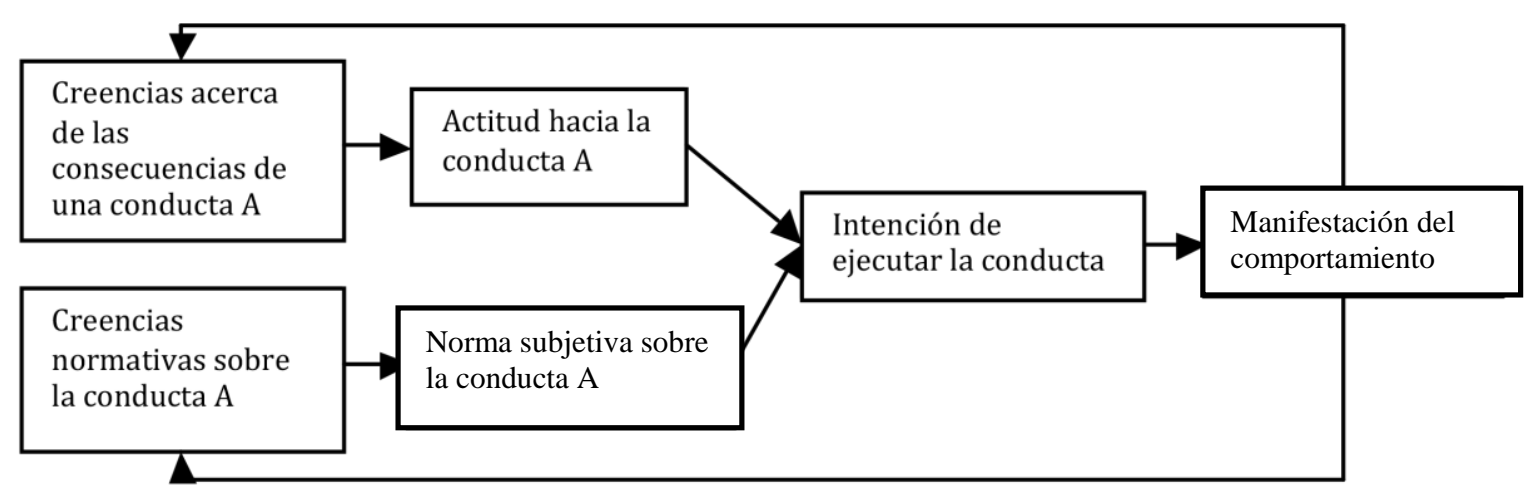

Figura 1. Modelo de Fishbein y Ajzen (1975)

Tanto los valores como las expectativas de Categoría 2.- Enfoque sociológico. Donoso éxito influyen a su vez sobre la persistencia en y Schiefelbein (2007) señalan que en forma la universidad. paralela al enfoque psicológico, se producen los

A continuación, en el Diagrama 2, podemos ver el modelo de Ethington (1990).

A modo de conclusión, los modelos psicológicos incorporan fundamentalmente variables individuales, esto es, características $\mathrm{y}$ atributos propios del estudiante, que dan cuenta, con diferente grado de ajuste, de la deserción o la persistencia. primeros modelamientos desde la perspectiva sociológica. Estos enfatizan la influencia de factores externos al individuo en la retención, los que se suman a los psicológicos. Entre estos modelos está la propuesta de Spady (1971) sobre deserción universitaria, quien desarrolla su modelo basado en la teoría del suicidio de Durkheim. Este último, sostiene que el suicidio es el resultado de la ruptura del individuo

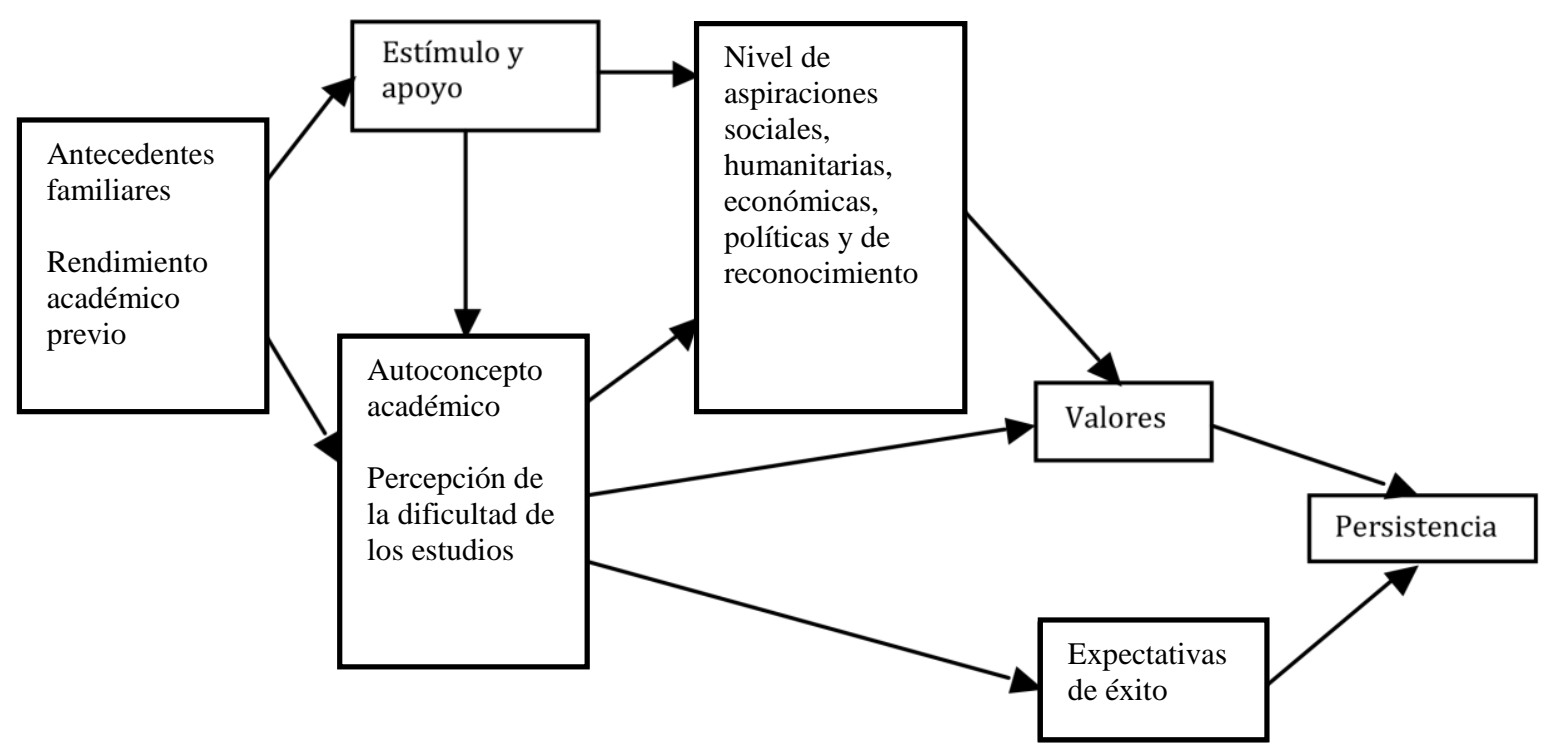

Figura 2. El modelo de Ethington (1990) 
con el sistema social por su imposibilidad de integrarse a la sociedad. La probabilidad de suicidio aumenta cuando existe una baja conciencia moral (congruencia normativa baja) y afiliación social insuficiente. Esto es, bajo apoyo de las relaciones sociales.

Spady (1971) sostiene que estos mismos tipos de integración afectan directamente la retención de los alumnos en la universidad. Sugiere que la deserción es el resultado de la falta de integración de los estudiantes en el entorno de la educación superior. Este autor también señala que el medio familiar es una de las principales fuentes que exponen a los estudiantes a influencias, expectativas y demandas, afectando su nivel de integración social en la universidad.

En el diagrama 3 se establece la relación que, según Spady (1971), el ambiente familiar influye sobre el potencial académico y la congruencia normativa.
Spady (1971) sostiene que existe una alta probabilidad de abandono de los estudios, cuando las diversas fuentes de influencia van en sentido negativo, lo que deriva en un rendimiento académico insatisfactorio, bajo nivel de integración social $y$, por ende, de insatisfacción y compromiso institucional. $\mathrm{Al}$ contrario, si los efectos van en dirección positiva y son congruentes con la situación inicial, el estudiante logra un desarrollo académico y social acorde tanto con sus propias expectativas como con las institucionales, lo que favorece significativamente su retención en la universidad.

Categoría 3.- Enfoque económico. Donoso (2007) señala que el enfoque económico responde a la aplicación del enfoque costobeneficio. Al respecto, sostiene que no siempre el racional de los beneficios opera en la forma esperada, esto es, cuando los beneficios sociales y económicos generados por los estudios universitarios son percibidos

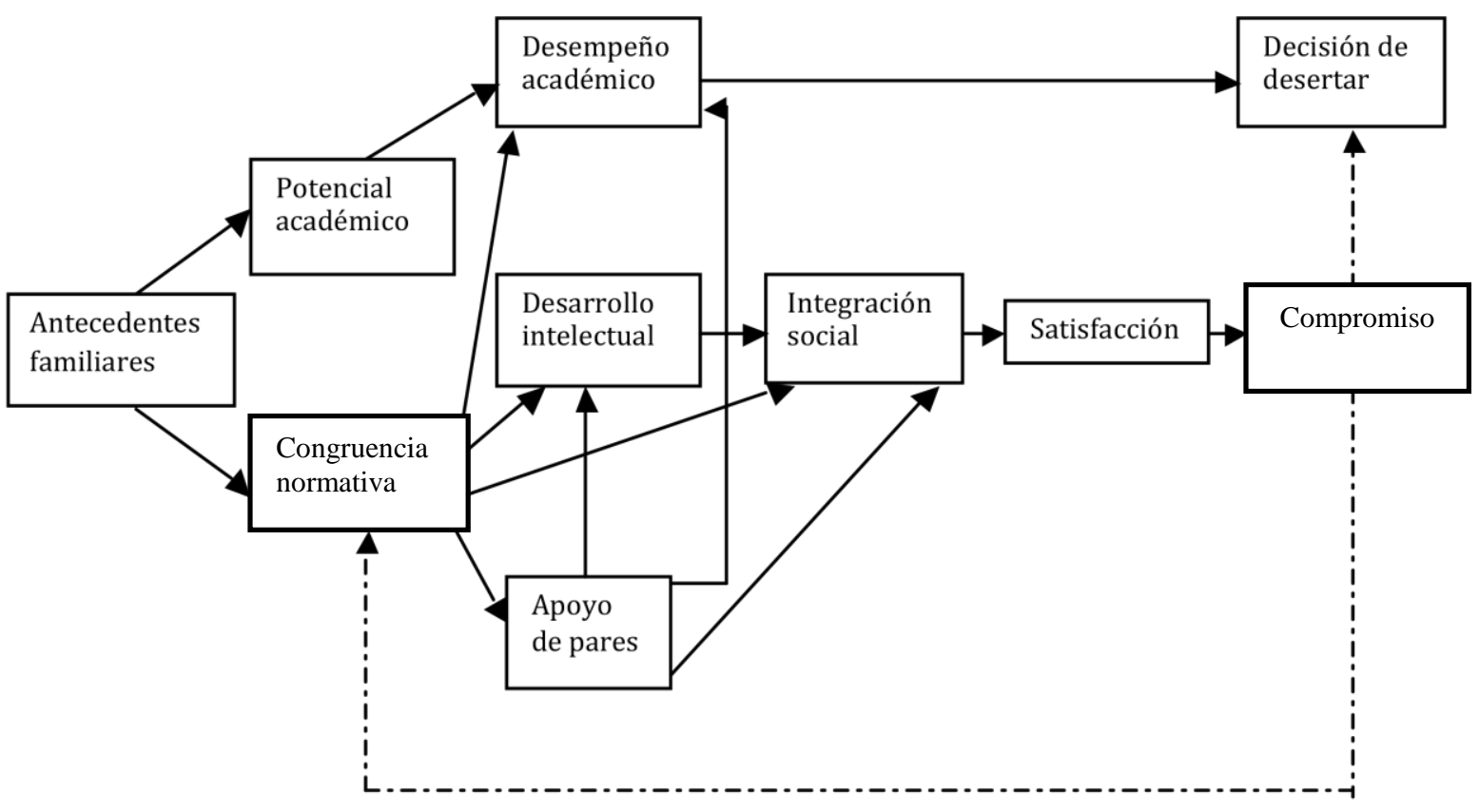

Figura 3. Modelo de Spady (1971) 
como inferiores a los derivados de actividades alternas, los sujetos optan por retirarse.

Himmel (2002) señala que este modelo busca privilegiar el impacto de los beneficios estudiantiles por sobre la deserción, dejando de lado las percepciones acerca de la adecuación de dichos beneficios a los costos de los estudios.

Las ayudas proporcionadas a los estudiantes en forma de becas constituyen un factor de peso en las posibilidades de permanencia, observándose que las tasas de deserción varían dependiendo de la cantidad y duración de la ayuda financiera con la que cuenta el estudiantado (Ishitani y DesJardins, 2003).

Categoría 4.- Enfoque organizacional. Donoso (2007) señala que en este caso se enfoca la deserción desde las características de la institución universitaria, atendiendo a los servicios que ofrece a los estudiantes. En estos modelos tienen un rol crucial las variables de calidad "de la docencia" y "de las experiencias de los estudiantes en el aula". A estas variables se suman -en oportunidades- los beneficios proporcionados por la organización a los estudiantes en salud, deportes, cultura y apoyo académico y docente (recursos bibliográficos, laboratorios y número de alumnos por docente). Estas variables pertenecen al ámbito operacional de las universidades, siendo completamente factible su intervención.

Este modelo de análisis sostiene que la deserción depende de las cualidades de la organización en la integración social, y más particularmente en el abandono de los estudiantes que ingresan a ella (Berger y Milem, 2000; Berger, 2002; Kuh, 2002, como se citó en Donoso \& Schiefelbein, 2007). En este enfoque, es altamente relevante la calidad de la docencia y de la experiencia de aprender en forma activa por parte de los estudiantes en las aulas, las cuales afectan positivamente la integración social del estudiante (Braxton et al., 1997).

“...como actores principales dentro de esos sistemas, las universidades públicas y privadas, han ingresado a una época de turbulencias para la cual no se prevé término. La actual encrucijada tiene su origen en un simple hecho: las demandas ejercidas sobre las universidades superan su capacidad de respuesta" (Clark, 1998, p. 129, como se citó en Rojas, 2009).

Categoría 5.- Enfoque interaccionista. En sociología, existe una larga tradición teórica y empírica que ha explorado aspectos organizacionales que impactan la retención estudiantil (Tinto 1975; Bean 1982, como se citó en Donoso \& Schiefelbein, 2007; Astin, 1984; Pascarella \& Terenzini 1991). Tinto es uno de los autores más reconocidos en este ámbito sugiriendo, entre otros, los efectos positivos de la interacción entre profesores y alumnos.

Tinto (1987, como se citó en Canales y De los Ríos, 2009) plantea que mientras mayor interacción entre alumnos y profesores exista, mayores probabilidades de que los alumnos finalicen sus estudios.

Centrar la atención en el contexto social e institucional resulta fundamental para comprender la retención, ya que permite comprender dicho fenómeno como el resultado de la interacción entre un estudiante y el medio en el cual se desenvuelve (Thomas, 2002, Tierney, 200, como se citó en Canales y De los Ríos, 2009).

Tinto (1975) explica el proceso de permanencia en la educación superior como una función del grado de ajuste entre el estudiante y la institución, adquirido a partir de las experiencias académicas y sociales 
(integración) (Díaz, 2008). El modelo de Tinto es el de mayor influencia sobre el estudio de la retención.

"Reconociendo el rol de las instituciones en la generación de los resultados, Tinto desarrolla un refinado modelo para explicar las decisiones de los estudiantes por permanecer o cambiarse de las instituciones de educación superior (Tinto desarrolla su modelo en los Estados Unidos). Este atraviesa por la integración social e intelectual de los estudiantes, aquellos que alcanzan esta condición tienen mayores posibilidades de permanecer en la institución" (Peterson, 1993, p. 660 como se citó en Canales \& De los Ríos, 2009).

Tinto incorpora al modelo de Spady la teoría de intercambio de Nye (1979, como se citó en Donoso, 2007) la cual asume como principio que las personas tienden a evitar las conductas que implican un costo de algún tipo para ellas y por ende buscan recompensas en las relaciones, interacciones y estados emocionales.

Según Tinto (1975), los estudiantes emplean "la teoría del intercambio" en la construcción de su integración social y académica. De esta forma, si los beneficios de permanecer en la institución son percibidos por los estudiantes como mayores que los costos personales (esfuerzo y dedicación, entre otros), entonces este permanecerá en la institución. Alternativamente, si se reconocen otras actividades como fuente de mayores recompensas, el estudiante tenderá a desertar.

Asimismo, Tinto reconoce, en sentido amplio, que la trayectoria de interacciones de la persona con los sistemas académico y social de la universidad puede derivar en su alejamiento de la institución.
En suma, mientras más se consolide el compromiso del estudiante con la obtención de su grado o título y con la institución, al mismo tiempo que mientras mejor sea su rendimiento académico e integración social, menos probable es que el alumno deserte.

Pese a todo lo señalado, los estudios con el modelo de Tinto no muestran resultados estables en términos del peso y el sentido de los factores postulados según diferentes tipos o modalidades institucionales. Algunas investigaciones confirman la capacidad predictiva directa de la integración social sobre la deserción en las instituciones de educación más tradicionales (Pascarella y Terenzini 1980).

Bean y Metzner (1985) asumen que los factores externos a la institución pueden ser de importancia, ya que impactan tanto las actitudes como las decisiones del estudiante durante su estadía en la institución. Investigaciones más recientes (Bean y Vesper 1990) señalan que factores no cognitivos, a saber: características personales (actitudes, aspiraciones, motivaciones, intereses), ambientales y organizacionales tienen un peso significativo en la deserción, en particular en la que es voluntaria.

Díaz Peralta (2008) encontró seis predictores de la deserción estudiantil en college's norteamericanos: integración académica, integración social, estado socioeconómico, género, calidad de la carrera y el promedio de notas en cada semestre. Otra teoría integrada en este modelo adaptativo es la del Agotamiento Estudiantil (Attrition) de Bean y Metzner (1985).

Todos los modelos reconocen que en el inicio de la carrera del estudiante, ocupan un lugar preciso las dimensiones motivacional y vocacional, y los efectos institucionales debidos a las condiciones de masificación de la 

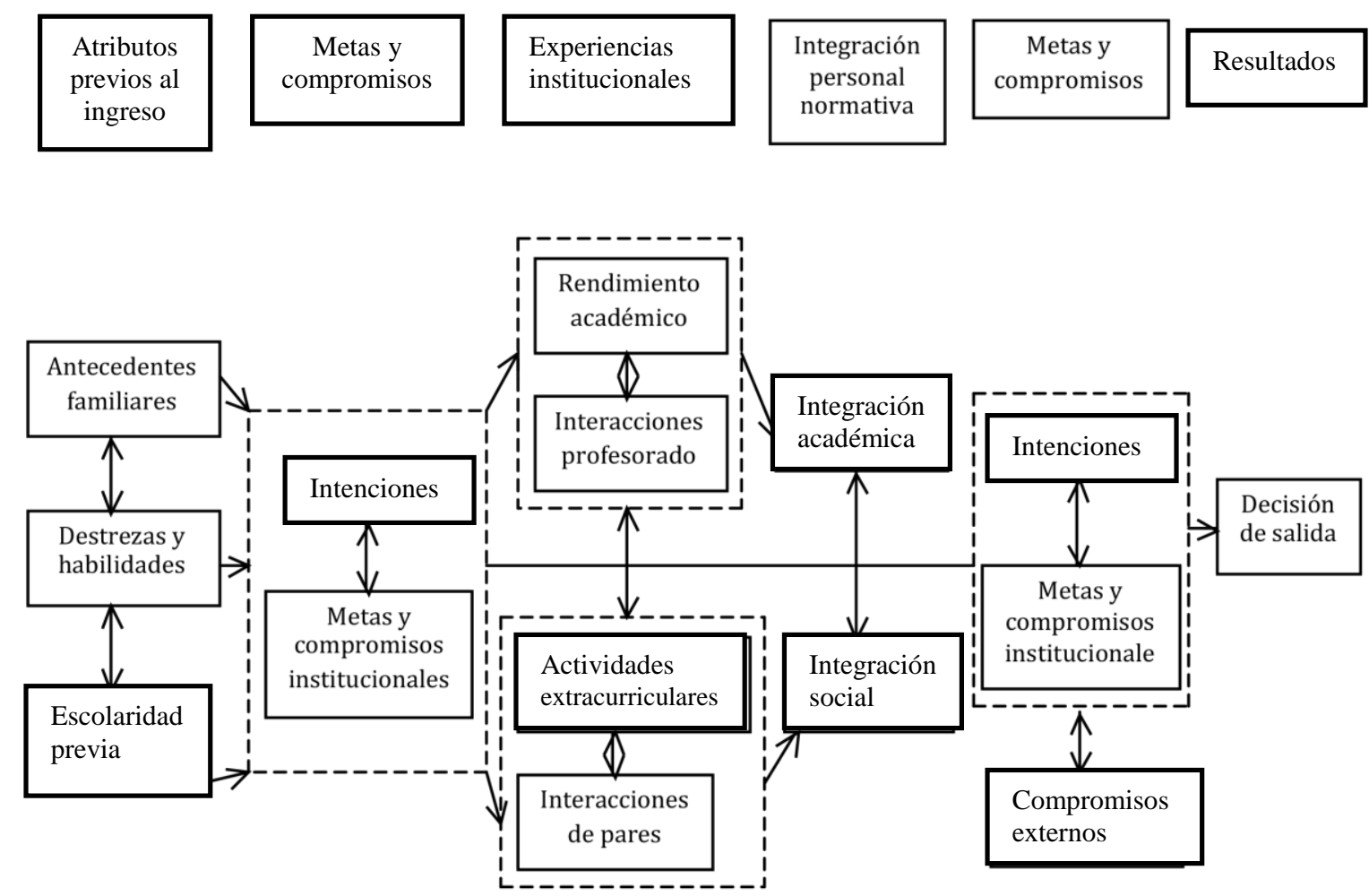

Figura 4. Modelo de Tinto (1987). Adaptado de Tinto (1975)

enseñanza y de equipamientos. Seguidamente, entienden que los factores personales y los de infraestructura pierden progresivamente incidencia y cobran importancia los de tipo social externo o como denominamos aquí 'extra-académicos' (Boado, 2003).

Pascarella (1985) sugiere un modelo causal general con consideraciones explícitas respecto de las características institucionales y ambientales. El autor sostiene que el desarrollo y cambio de los estudiantes es función de cinco conjuntos de variables, dos de las cuales están relacionadas con antecedentes y características personales a saber: aptitudes, rendimientos, personalidad, aspiraciones y etnicidad. El segundo grupo lo conforman las características estructurales y organizacionales de la institución, admisión, estudiantes de la facultad, selectividad, porcentaje de residentes. Este grupo se relaciona con el tercer conjunto de variables: entorno institucional. Estos tres grupos de características incluyen, en turnos, al cuarto grupo que considera el conjunto de variables asociadas a la frecuencia y contenido de las interacciones con los miembros de la facultad y los pares. El quinto grupo se refiere a la calidad del esfuerzo desplegado por el estudiante por aprender (Pascarella y Terenzini, 1991).

El modelo de Nora (1990), en Donoso \& Schiefelbein (2007), relaciona las habilidades académicas de los estudiantes al ingresar a la universidad, las necesidades de apoyo financiero, los beneficios estudiantiles de la institución y el desempeño académico de los alumnos en ella, como factores determinantes de la deserción.

Cabrera, Castañeda, Nora y Hegnstler (1992), en Donoso \& Schiefelbein (2007), plantean que la persistencia en la educación superior se modela mediante un proceso que abarca tres etapas. 


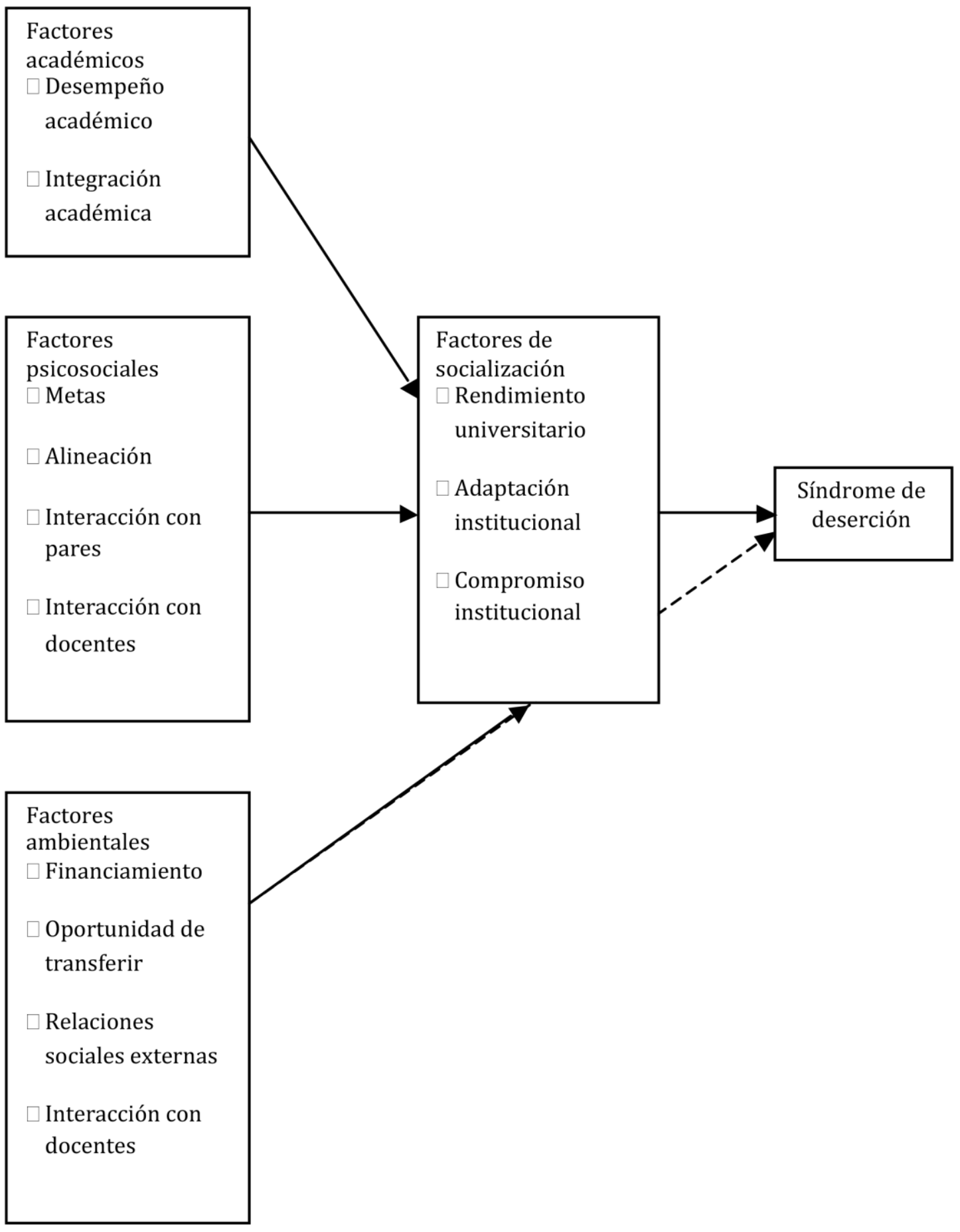

Figura 5. Modelo de Bean (1985)

Una primera etapa donde la base estudios en una institución, lo que implica que académica previa a la universidad y los factores socioeconómicos afectan al estudiante para continuar sus estudios universitarios.

La segunda etapa en la que el estudiante al ingresar lo hace con un cierto compromiso inicial con la institución, el que es revisado con el transcurrir del tiempo.

En la tercera etapa, cursando estudios "estima" los costos y beneficios asociados de sus universitarios, otros factores como las 


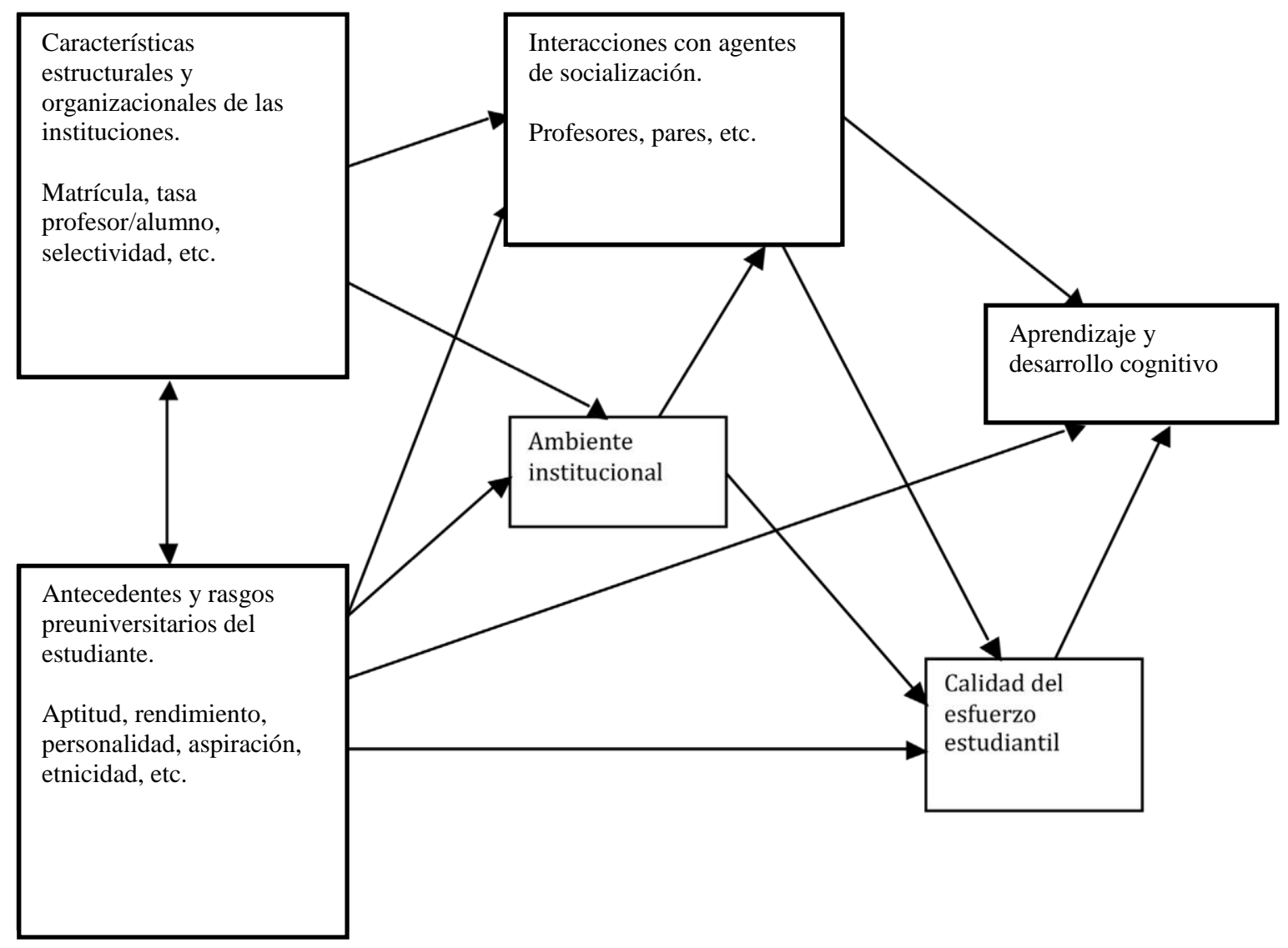

Figura 6. Modelo de Pascarella y Terenzini (1985)

experiencias académicas y sociales a las que se va exponiendo y su desempeño académico va cobrando fuerza. De este modo, las experiencias sociales y académicas positivas $y$ un desempeño académico adecuado reforzarían las percepciones acerca de los beneficios económicos y de otra naturaleza, que derivan como consecuencia de la culminación de los estudios. Por otra parte, los apoyos financieros y una adecuada integración académica influirían positivamente en la decisión de permanecer en la institución, al mantenerse de este modo el equilibrio entre el costo de cursar una carrera postsecundaria y el beneficio de obtener un título o grado académico. En cambio, las experiencias negativas, tales como las alzas en los valores de la matrícula o experiencias académicas

insatisfactorias, producen un desequilibrio entre el costo y el beneficio, incrementando la probabilidad de la deserción estudiantil.

\section{Antecedentes de instituciones para evitar la deserción}

Cabreraetal.(2006) señalanque debidoalretraso y abandono de los estudios, se implementaron los cursos cero (conocidos también como cursos de nivelación) por algunas universidades.

Grosset (1991) afirma que habría que mirar la deserción como un factor de eficiencia institucional y, como tal, las estrategias para afrontarla deberían contemplar tanto los procesos educativos como las características de los estudiantes que propician el abandono, pues la deserción está más en función de lo que ocurre después de entrar a la universidad, que lo que la precede. 
Se asume, también, en estos procesos la fuerte diferencia entre la concepción y el diseño, y la aplicación de un programa de retención en el, a menudo, rígido laberinto de las estructuras institucionales. Algunas consideraciones al respecto son que los programas de retención exitosos son más frecuentemente de carácter longitudinal (como los programas que la DPA [Dirección de Prospección Académica de la Universidad Peruana de Ciencias Aplicadas (UPC)] ha diseñado para los postulantes aprobados); que están integrados de manera paralela con los procesos de admisión $y$, finalmente, que su aplicación involucra generalmente a un amplio espectro de actores institucionales (Tinto, 1982).

La universidad responde institucionalmente al fenómeno de la deserción estudiantil, pero de una manera desarticulada pues no existe una construcción problémica del tema que permita la implementación de políticas y acciones que de manera sistemática enfrenten el abandono (Rojas, 2009).

\section{CONCLUSIONES}

Sabemos que existe una brecha académica entre lo que se enseña en las escuelas y lo que se exige en las universidades. Este no es un problema nuevo ni exclusivo del ámbito académico ni propio del Perú. Es más bien un viejo problema que tiene muchas variables y que, además, se da en todo el mundo. Esta brecha académica contribuye, entre otras variables, con la deserción estudiantil universitaria.

Un estudiante que no termina sus estudios universitarios es muy probable que sea subempleado obteniendo ingresos muy por debajo de los esperados. Esto genera frustración no solo en él sino en su entorno más cercano.

Algunas autoridades universitarias han tomado el problema de la deserción como propia y en varias universidades se ha logrado disminuir esa brecha académica creando programas o cursos que sirven para nivelar a los estudiantes recién ingresados a ella. Incluso, se dictan cursos de métodos o estrategias de aprendizaje de modo que faciliten su inserción a la vida universitaria. Adicionalmente, algunas universidades tiene asignado un equipo docente para la atención de posibles casos de deserción.

Las autoridades universitarias deben "tomar al toro por las astas", pues la deserción estudiantil es tarea de todos.

\section{REFERENCIAS}

Astin, A. W. (1984). Student Involvement: A Developmental Theory for Higher Education. Journal of College Student Development, 40(5), 518530. Recuperado de https://www. middlesex.mass.edu/ace/downloads/ astininv.pdf

Attinasi, L. C. (1986). Getting in: Mexican American Students' perceptions of their college-going behavior with implications for their freshman year persistence in the University. ASHE, 1986 Annual Meeting Paper. San Antonio, TX, EE. UU. (ERIC N. 268 869). Bean, J. \& Metzner, B. (1985). A Conceptual Model of Nontraditional Undergraduate Student Attrition. Review of Educational Research, 55(4), 485-540.

Bean, J., \& Vesper, N. (1990). Quantitative Approaches to Grounding Theory in Data: Using LISREL to Develop a Local Model and Theory of Student Attrition. In Annual Meeting AERA, April 17, 1990. Boston, EE.UU.

Bethencourt, J., Cabrera, L., Hernández, J., Álvarez, P., \& González, M. (2008). Variables psicológicas y educativas 
en el abandono universitario. Revista Electrónica de Investigación Psicoeducativa, 6(3), 603-622.

Boado, M. (2003). Una aproximación a la deserción estudiantil universitaria en Uruguay. Montevideo. Instituto Internacional para la Educación Superior en América Latina y el Caribe.

Braxton, J., Shaw-Sullivan, A. \& Johnson, R. (1997). Appraising Tinto's Theory of College Student Departure. En M. Paulsen (Ed.). Higher Education: Handbook of Theory and Research, Vol.12, (pp.107-495). Dordrecht: The Netherlands. Springer.

Cabrera, L., Bethencourt, J. T., Álvarez Pérez, P., \& Gónzalez Alfonso, M. (2006). El problema del abandono de los estudios universitarios. Relieve, 12(2), 171-203.

Canales, A., \& De los Ríos, D. (2009). Retención de estudiantes universitarios vulnerables en la educación universitaria. Calidad en la Educación, 30, 49-83.

Castaño, E., Gallón, S., Gómez, K., \& Vásquez, J. (2004). Deserción estudiantil universitaria: una aplicación de modelos de duración. Lecturas de Economía, 60, 39-65.

Corominas, E. (2001). La transición de los estudios universitarios. Abandono o cambio en el primer año de universidad. Revista de Investigación Educativa, 19(1), 127-152.

De Miguel, M. \& Arias, J. (1999). La evaluación del rendimiento inmediato en la enseñanza universitaria. Revista de Educación, 320, 353-377.

Del Moral, A., Pazos, J., Rodríguez, E., Rodríguez-Patón, A., \& Suárez, S. (2007). Gestión del Conocimiento. España: Thomson.

Díaz-Peralta, C. (2008). Modelo conceptual para la deserción estudiantil universitaria chilena. Estudios Pedagógicos, 34(2), 65-86. Donoso,S., \&Schiefelbein,E.(2007).Análisis de los modelos explicativos de retención de estudiantes en la universidad: una visión desde la desigualdad social. Estudios Pedagógicos, 33(1), 7-27.

Durán, J. \& Díaz, G. (1990). Análisis de la deserción estudiantil en la Universidad Autónoma Metropolitana. Revista de Educación Superior, 19(74), Recuperado de: http://publicaciones. anuies.mx/revista/74

Eccles, J.S., \& Wigfield, A. (2002). Motivational Belief, values and goals. Annual Review of Psychology, 53, 109-132.

Ethington, C. A. (1990). A psychologycal model of student persistence. Research in Higher Education, 31(3), 266-269.

Fishbein, M., \& Ajzen, I. (1975). Belief, attitude, intention and behavior: An introduction to theory and research. California.: Addison Wesley Pub.

Giovagnoli, P. (2002). Determinantes de la deserción y graduación universitaria: Una aplicación utilizando modelos de duración. Documento de trabajo N. 37, 2002, Universidad Nacional de la Plata, Argentina.

González, D. \& Girón, L. (2005). Determinantes del rendimiento académico y la deserción estudiantil en el programa de Economía de la Pontificia Universidad Javeriana de Cali. Economía, Gestión y Desarrollo, 3, 173-201.

Grosset, J.M. (1991). Patterns of integration, commitment, and student characteristics, and retention among younger and older students. Research in Higher Education, 32(2), 159178. http://dx.doi.org/10.1007/ BF00974435 
Himmel, E. (2002). Modelos de Análisis de la Deserción Estudiantil en la Educación Superior. Calidad en la Educación, 17, 91-107.

Ishitani, T. T. y DesJardins, S. L. (2003). A longitudinal investigation of dropout from college of the United States. Journal of College Student Retention: Research, Theory and Practice, 4(2), 173-201.

MINISTERIO DE EDUCACIÓN NACIONAL (2006). Educación superior en Colombia. Bogotá: Ministerio de Educación Nacional.

Pascarella, E., \& Terenzini, P. (1980). Predicting freshmen persistence and voluntary dropouts decisions from a theoretical model. Journal of Higher Education, 51(1), 60-75.

Pascarella, E., \& Terenzini, P. (1991). How college affects students. San Francisco: JosseyBass.

Picardo, O., Escobar, J., \& Balmore, R. (2004). Diccionario Enciclopédico de Ciencias de la Educación. 1a Ed. San Salvador: Centro de Investigación Educativa, Colegio García Flamenco.

Rivera, E., Roca, H., Echart, B., Alfaro, E., López, A., Farfán, S., Barrera, B. (2005). Estudio sobre la repitencia y deserción en la educación superior en Bolivia. Secretaría Ejecutiva de Desarrollo Universitario, Universidad Autónoma Juan Misael Saracho, IESALC/UNESCO, Tarija. En www.iesalc.unesco.org.ve

Rojas, M. (2009). El Abandono de los Estudios: Deserción y Decepción de la Juventud. Hologramática. Facultad de Ciencias Sociales de la Universidad Nacional de Lomas de Zamora, Argentina. Número 10, V4ISSN 1668-5024, 75-94. Recuperado de http://www.cienciared. com.ar/ra/doc.php?n=1081

Simpson, S. (2004). A Study of Attrition in Higher Education With Implications For
Supportive Services. Tesis presentada para graduarse de la Universidad de Marshall.

Spady, W. (1971). Dropouts from higher education: toward an empirical model. Interchance, 2, 38-62. http://dx.doi. org/10.1007/BF02282469

Tejedor, F., \& García-Valcárcel, A. (2007). Causas del bajo rendimiento del estudiante universitario (en opinión de los profesores y alumnos). Propuestas de mejora en el marco del EEES. Revista de Educación, 342, 443-473.

Tinto, V. (1975). Drop out from Higher Education: A Theoretical Synthesis of Recent Research. Review of Educational Research, 45(1), 89-195.

Tinto, V. (1982). Definir la Deserción: Una Cuestión de Perspectiva. Revista de Educación Superior, 71, 33-51.

Tinto, V. (1987). The Principles of Effective Retention. Paper presented at the Fall Conference of the Maryland College Personnel Association.

Tinto, V. (1989). Misconceptions Mar Campus Discussions of Student Retention. The Chronicle of Higher Education; Sep. 6, 1989; 36, 1; Academic Research Library.

Tinto, V. (2003). Promoting Student Retention Through Classroom Practice; Presented at Enhancing Student Retention: Using International Policy and Practice. An international conference sponsored by the European Access Network and the Institute for Access Studies at Staffordshire University. Amsterdam, November 5-7.

Tinto, V. (2006). Research and Practice of Student Retention: What Next? Journal of College Student Retention, 8(1), 1-19.

UNESCO, I. (2007). Informe sobre la educación superior en América Latina y el Caribe 2000-2005. La metamorfosis de la 
educación superior. Caracas, enero de 2007.

Vivas, J. (2005). El abandono de estudiantes universitarios: análisis y reflexiones sobre la experiencia sobre la Universidad Autónoma de Barcelona. Trabajo presentado en el Encuentro Internacional Deserción Estudiantil en Educación Superior. Experiencias Significativas. Bogotá: Colombia.

(C) Los autores. Este artículo es publicado por la Revista Digital de Investigación en Docencia Universitaria del Área de Investigación de la Dirección de Calidad Educativa, Universidad Peruana de Ciencias Aplicadas. Este es un artículo de acceso abierto, distribuido bajo los términos de la Licencia Creative Commons Atribución-CompartirIgual 4.0 Internacional.( http://creativecommons.org/licenses/by-sa/4.0/), que permite el uso no comercial, distribución y reproducción en cualquier medio, siempre que la obra original sea debidamente citada. 
